\title{
Association between a progesterone receptor mutation and hepatitis E sero-positivity in liver transplant recipients
}

\author{
Jose D. Debes MD ${ }^{1,2}$ (1) | Zwier M. A. Groothuismink ${ }^{2}$ | Robert A. de Man MD, $\mathrm{PhD}^{2}$ | \\ Andre Boonstra $\mathrm{PhD}^{2}$
}

${ }^{1}$ Department of Medicine, University of Minnesota, Minneapolis, Minnesota

${ }^{2}$ Department of Gastroenterology and Hepatology, Erasmus MC, University Medical Center, Rotterdam, The Netherlands

\section{Correspondence}

Jose D. Debes, MD, and Andre Boonstra, PhD, Department of Gastroenterology and Hepatology, Erasmus MC, University Medical Center Rotterdam, Wytemaweg 80, Room Na1011, 3015 CE Rotterdam, The Netherlands. Email: debes003@umn.edu (J.D. D.) and p.a.boonstra@erasmusmc.nl (A. B.)

Funding information

American College of Gastroenterology, Grant/Award Number: ACG-CR-033-2016;

Robert Wood Johnson Foundation

\begin{abstract}
Problem: We investigated if the PROGINS mutation increases the risk of hepatitis E virus (HEV) infection in liver transplant recipients. PROGINS was analyzed through KASP assay; HEV serologies assessed via enzyme-linked immunosorbent assay and multiplex cytokine assays were evaluated in plasma with the ProcartaPlex human immunoassay. Seventy liver transplant recipients were evaluated, of which 23 (33\%) were HEV immunoglobuln G (IgG)-positive (HEV+). The frequency of PROGINS in the HEV+ group was 34\%, compared with $14 \%$ in those that were HEV IgG negative (HEV-). Cytokine measurements in a sub-set of samples from HEV+/PROGINS+ individuals showed decreased plasma levels of monokine induced by gamma interferon, a proliferation-inducing ligand, and stem cell factor, as well as increased levels of eotaxin-3 and interleukin-31 compared with those HEV-/PROGINS- samples. Our findings suggest an association between the PROGINS mutation and seropositivity for HEV in liver transplant recipients with consequent distorted cytokine levels.

KEYWORDS

cytokines, HEV, liver transplant, PROGINS
\end{abstract}

\section{1 | INTRODUCTION}

Infection with the hepatitis E virus (HEV) can cause acute hepatitis in immunocompetent hosts as well as liver failure in pregnant women, and can lead to chronic infection or acute reactivation, the latter as case reports, in solid organ transplant recipients. ${ }^{1-3}$ Overall, the understanding of mechanisms and host factors that affect prevalence or predispose to HEV infection in individuals with immune-suppression or undergoing pregnancy is limited. ${ }^{4}$ Moreover, whether risk factors that predispose to viral infection in these two conditions are interrelated remains undefined. Interestingly, a study by Bose et al ${ }^{5}$ described a mutation in the progesterone receptor (PR) named PROGINS to be associated with acute viral hepatitis and fulminant hepatic failure secondary to HEV in pregnant women. During pregnancy, progesterone exerts an important effect on peripheral lymphocytes expressing PR to modulate uterine immunity. The PROGINS mutation, which depending on the specific ethnicity can affect $2 \%$ to $14 \%$ of the population, consists of two single nucleotide polymorphism (SNP) with a G-intron insert, and past studies have demonstrated the mutation to affect the functionality of the PR. ${ }^{6,7}$ It is theorized that progesterone could have a differential effect on peripheral lymphocytes expressing PR in individuals with the mutation, therefore predisposing them to HEV infection. ${ }^{8}$ Our team recently showed that the PROGINS mutation is expressed with a higher frequency in individuals with human immunodeficiency virus (HIV) who are seropositive for HEV and that the mutation conferred alterations in peripheral immune markers as well as a decreased T-cell response. That study showed for the first time that the mutation in the PR could confer high risk for HEV in a nonpregnant immune-suppressed cohort. Since HEV can reactivate or lead to chronic hepatitis in solid-organ transplant recipients more frequently than in other populations, we thought to determine the presence of PROGINS in liver transplant recipients and its association to $\mathrm{HEV}$ infection. 


\section{2 | MATERIALS AND METHODS}

\section{1 | Samples}

We evaluated plasma samples from 70 individuals who received a liver transplantation and analyzed them for the presence of PROGINS Samples were collected between 2000 and 2015 and kept at $-80^{\circ} \mathrm{C}$. Median age at collection was 58 years (interquartile range [IQR] 41-65) and $27 \%$ were females. A group of 187 healthy samples previously described were used as controls. ${ }^{9}$ We obtained approval for our study by the Medical Ethical Review Board of the Erasmus Medical Center (reference number MEC- 2011-438).

\section{2 | Hepatitis E virus and single nucleotide polymorphism determination}

For detection of HEV specific immunoglobulin $M$ and immunoglobulin G (IgG) we used the commercially available enzyme-linked immunosorbent assay (ELISA) test (Wantai, China) according to the manufacturer's instructions. For genotyping, competitive allele-specific polymerase chain reaction assays (KASP; LGC genomics, Huddleston, UK) were employed. The PROGINS allele was defined by the exon 4 missense SNP rs1042838, Val660Leu, as previously described. ${ }^{10}$ Plasma samples stored at $-80^{\circ} \mathrm{C}$ were used for DNA extraction and genotyping procedures which were carried out at LGC genomics using $\geq 5 \mathrm{ng}$ of genomic DNA. ${ }^{11}$

\section{3 | Cytokine assessment}

For the assessment of cytokines/chemokines and other plasma proteins, we performed multi-analyte profiling using the ProcartaPlex human immunoassay (Affymetrix, eBioscience, Austria). The panel measured APRIL, BAFF, eotaxin-3 (CCL26), FGF-2, G-CSF, GRO- $\alpha$, interleukin-1 $\alpha$ (IL-1 $\alpha$ ), IL-1 $\beta$, IL-21, IL-22, IL-23, IL-3, monokine induced by gamma interferon (MIG) (CXCL9), stem cell factor (SCF), tumor necrosis factor- $\alpha$, IL-8, IL-31, LIF, MIP-3 $\alpha$, TRAIL, TWEAK, and vascular endothelial growth factor-A. The assay was conducted according to the manufacturer's instructions, identical to the procedures used in our previous studies. ${ }^{12}$ All samples were analyzed in one run. Statistical analyses: During statistical analyses, baseline characteristics were compared between HEV IgG positive and negative patients expressing PROGINS mutation or wild type PR. In general, quantitative comparisons were performed using the parametric the Student $t$ test (for normally distributed values) and the nonparametric Wilcoxon test or Mann-Whitney tests (for values that did not show a normal distribution). A $P<.05$ was considered statistically significant.

\section{3 | RESULTS}

Of the 70 liver transplant recipients, 47 (67.1\%) were HEV IgGnegative (HEV-) and 23 (32.8\%) HEV IgG-positive (HEV+) as assessed by ELISA. We found the frequency of PROGINS in HEV+ to be $34.7 \%(\mathrm{~N}: 8)$, compared with $14.7 \%(\mathrm{~N}: 7)$ in those that were HEV- (RR, 2.2; confidence interval, 0.9-5.5; $P=.01$; Figure 1). Interestingly, the HEV- liver transplant recipient cohort showed a similar frequency of PROGINS as that of our control group (14\% each), suggesting that indeed the mutation could confer a risk factor for an altered immune response towards the virus upon exposure. Demographics between both groups ( $\mathrm{HEV}-$ and $\mathrm{HEV}+$ ) were similar, with median age of both groups at 57 years (IQR, 47-62 and 41-64, respectively) and a similar proportion of males $(70 \%$ and $68 \%$, respectively), suggesting that neither factor played a role in this setting. Almost half of the HEV+ patients (10/23) became seropositive following liver transplantation (median time 3 years; IQR, 2-7). In this group, $50 \%$ ( $N: 5)$ had the presence of the PROGINS mutation, further strengthening the concept of an association between HEV-seropositivity and the PROGINS mutation in liver transplant recipients. The specific route of $\mathrm{HEV}$ transmission in this cohort could not be established.
(A)

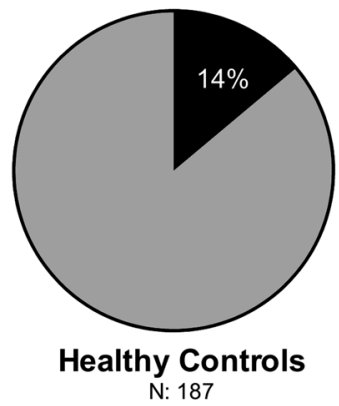

(B)

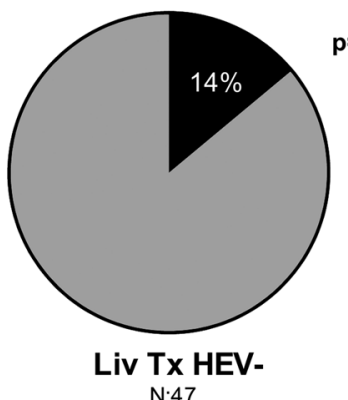

(C)

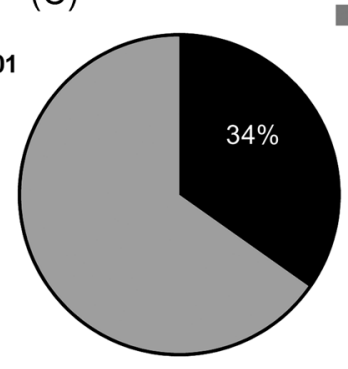

Liv Tx HEV+

$\mathrm{N}: 23$

FIGURE 1 Pie charts showing frequency of PROGINS as a percentage in (A) healthy controls (N:187); (B) liver transplant recipients HEV- (N:47), and (C) liver transplant recipients HEV+ (N:23). Dark color indicates presence of PROGINS mutation and gray color indicates wildtype progesterone receptor. $P$ value compares difference in PROGINS frequency between liver transplant recipients HEV- and HEV+. HEV, hepatitis $\mathrm{E}$ virus 
Next, we performed multi-analyte profiling of 22 immune markers in plasma in a subgroup of HEV+ and HEV- liver transplant recipients who expressed the PROGINS mutation and a group of HEV- recipients without the PROGINS mutation ( $N: 7$ in each group). We found increased levels of eotaxin-3 (CCL26) and IL-31, both predominantly implicated in Th2 dominated responses, and reduced plasma levels of MIG; CXCL9, APRIL, and SCF in the HEV+/PROGINS+ individuals when compared with HEV- liver transplant recipients regardless of mutation status (Figure 2). MIG, APRIL, and SCF are chemotactic and play a role in recruiting cells during inflammatory responses, or directly affect the function of T and B cells. ${ }^{13}$ From these proteins only SCF $(P=.01)$, and APRIL $(P=.02)$ showed statistical significance, while IL-31 showed a $P=.05$. In our cohort, only three individuals developed chronic HEV infection with one of them expressing the PROGINS mutation. With such a low number of subjects we cannot hypothesize on the role of PROGINS in the progression towards HEV chronicity.

\section{4 | DISCUSSION}

Our group recently described that the presence of the PROGINS mutation induced an impaired immune response in HIV-positive individuals and was associated with HEV-seropositivity in such patients. ${ }^{9}$
We then hypothesized that a "dual immune hit" represented by the mutation coupled with that induced by HIV could abrogate the response towards HEV exposure. In the current study, we found that a similar effect could occur in liver transplant recipients.

$\mathrm{HEV}+$ patients with PROGINS mutation expressed altered plasma levels of multiple immune markers when compared with those without the mutation. The observed alterations in plasma levels in $\mathrm{HEV}+$ patients with PROGINS mutation are suggestive of weaker antiviral immune responses in the group since the levels of Th2associated proteins IL-31 and eotaxin-3 were enhanced, while the proinflammatory markers MIG, APRIL reduced. ${ }^{13,14}$ The high frequency of the PROGINS mutation in the HEV+ patients combined with altered plasma cytokine profile warrant further detailed studies on the concept of an impaired immune response secondary to the presence of the mutation. However, caution should be used when interpreting these findings as they represent a one-time crosssectional measurement in a small sub-group of individuals. Moreover, a recent study performed entirely in an HIV-population suggests an inverse correlation between PROGINS and HEV infection. ${ }^{15}$ In addition, it is possible that cytokine levels are secondary to HEV serostatus, and that the cytokines are affected by immunosuppressive drugs administered to prevent graft rejection. Finally, only two of the proteins showed statistical significance, although the trend among the others was evident. With our low number of samples and
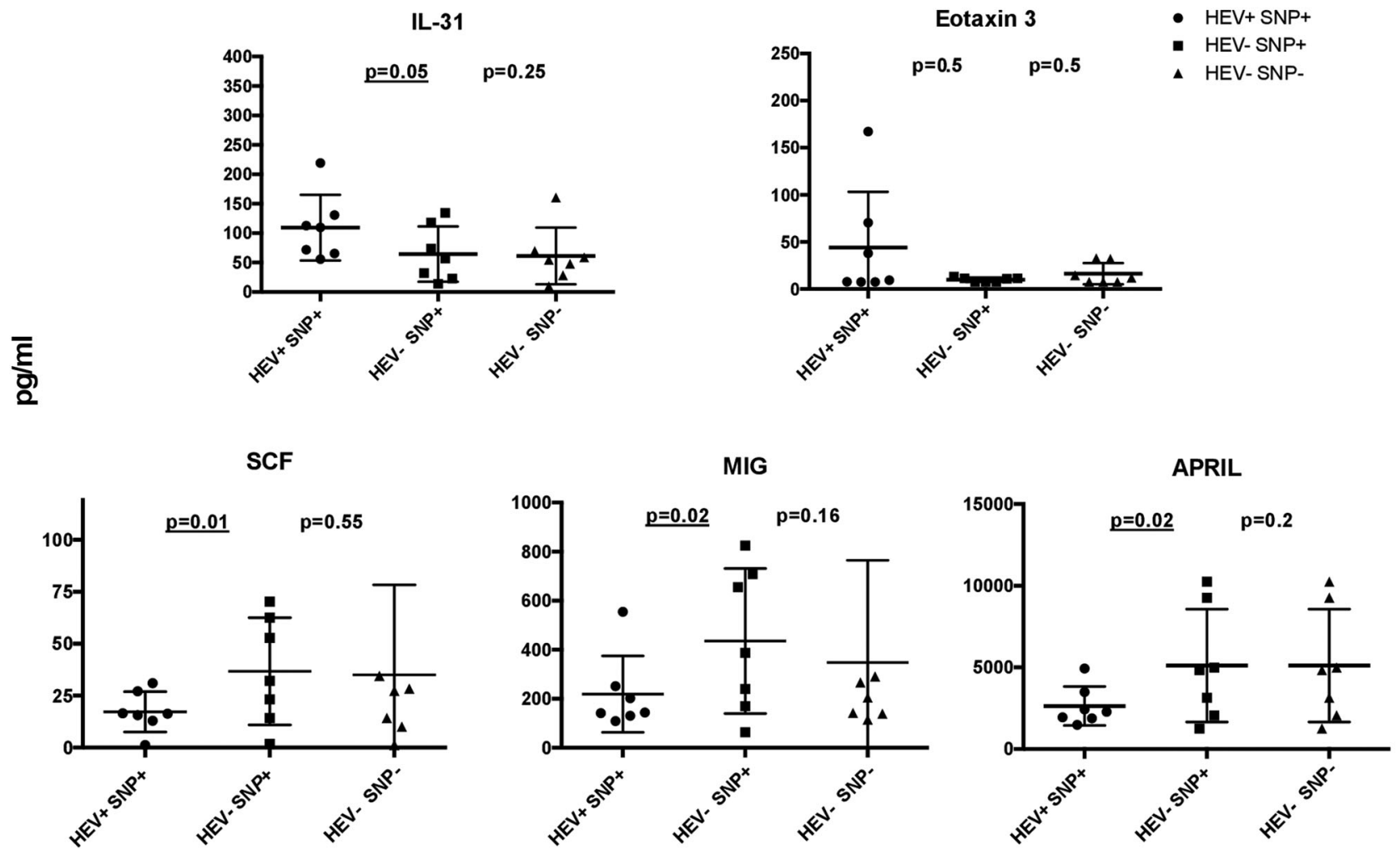

FIGURE 2 Plasma levels of IL-31, Eotaxin 3, SCF, MIG, and APRIL in 21 individuals (seven HEV+/SNP+, seven HEV-/SNP+, and seven HEV -/SNP-), measured with multiplex ELISA. $Y$-axis represents levels in $\mathrm{pg} / \mathrm{mL} ; X$-axis represents the different groups. $P$ values represent comparison between HEV-/SNP- and HEV+/SNP+. APRIL, a proliferation-inducing ligand; ELISA, enzyme-linked immunosorbent assay; HEV, hepatitis E virus; IL, interleukin; MIG, monokine induced by gamma interferon; SCF, stem cell factor; SNP, single-nucleotide polymorphism 
common variations of cytokines in blood, these specific findings should be addressed in a larger cohort before solid conclusions are drawn. In summary, our results show an association with the PROGINS mutation and HEV seroprevalence in liver transplant recipients. A larger validation study, as well as a study involving other solid organ transplant populations will be necessary to further understand the role of PROGINS in this setting. Our findings albeit from a small cohort, suggest an association between seropositivity for HEV and the PROGINS mutation in liver transplant recipients. This association correlated with alternations in peripheral cytokine levels, further strengthening the concept of an immune-related effect.

\section{ACKNOWLEDGMENTS}

Supported by the American College of Gastroenterology (ACG-CR033-2016), Robert Wood Johnson Foundation (AMFDP), and NIH$\mathrm{NCl}$ R21 CA215883-01A1 all to JDD.

\section{CONFLICT OF INTERESTS}

The authors declare that there are no conflict of interests.

\section{ORCID}

Jose D. Debes (D) http://orcid.org/0000-0002-1512-2604

\section{REFERENCES}

1. Abravanel F, Lhomme S, Chapuy-Regaud S, et al. Hepatitis E virus reinfections in solid-organ-transplant recipients can evolve into chronic infections. J Infect Dis. 2014;209(12):1900-1906.

2. Kamar N, Selves J, Mansuy JM, et al. Hepatitis E virus and chronic hepatitis in organ-transplant recipients. N Engl J Med. 2008;358(8): 811-817.

3. le Coutre P, Meisel H, Hofmann J, et al. Reactivation of hepatitis $E$ infection in a patient with acute lymphoblastic leukaemia after allogeneic stem cell transplantation. Gut. 2009;58(5):699-702.

4. Debes JD, Pisano MB, Lotto $M$, Re V. Hepatitis $E$ virus infection in the HIV-positive patient. J Clin Virol. 2016;80:102-106.

5. Bose PD, Das BC, Kumar A, Gondal R, Kumar D, Kar P. High viral load and deregulation of the progesterone receptor signaling pathway: association with hepatitis E-related poor pregnancy outcome. J Hepatol. 2011;54(6):1107-1113.

6. Romano A, Delvoux B, Fischer DC, Groothuis P. The PROGINS polymorphism of the human progesterone receptor diminishes the response to progesterone. J Mol Endocrinol. 2007;38(1-2):331-350.

7. Modugno F. Ovarian cancer and polymorphisms in the androgen and progesterone receptor genes: a HuGE review. Am J Epidemiol. 2004; 159(4):319-335.

8. Sivils JC, Storer CL, Galigniana MD, Cox MB. Regulation of steroid hormone receptor function by the 52-kDa FK506-binding protein (FKBP52). Curr Opin Pharmacol. 2011;11(4):314-319.

9. Debes JD, Pas SD, Groothuismink ZMA, van der Ende ME, de Man RA, Boonstra A. A mutation in the progesterone receptor predisposes to HEV infection in HIV-positive patients. Liver Int. 2018;38(5):792-796.

10. Pearce $\mathrm{CL}$, Hirschhorn JN, Wu AH, et al. Clarifying the PROGINS allele association in ovarian and breast cancer risk: a haplotype-based analysis. J Natl Cancer Inst. 2005;97(1):51-59.

11. Verstrepen BE, de Groot NG, Groothuismink ZM, et al. Evaluation of IL-28B polymorphisms and serum IP-10 in hepatitis C infected chimpanzees. PLoS One. 2012;7(10):e46645.

12. Spaan M, Claassen MA, Hou J, Janssen HL, de Knegt RJ, Boonstra A. The intrahepatic $T$ cell compartment does not normalize years after therapy-induced hepatitis C virus eradication. J Infect Dis. 2015; 212(3):386-390.

13. Zlotnik A, Yoshie O. The chemokine superfamily revisited. Immunity. 2012;36(5):705-716.

14. Tangye SG, Bryant VL, Cuss AK, Good KL. BAFF, APRIL, and human B cell disorders. Semin Immunol. 2006;18(5):305-317.

15. López-López $P$, Rivero-Juarez $A$, Frias $M$, et al. Mutations in the progesterone receptor (PROGINS) may reduce the symptoms of acute hepatitis E and protect against infection. Front Microbiol. 2019; 10:2617.

How to cite this article: Debes JD, Groothuismink ZMA, de Man RA, Boonstra A. Association between a progesterone receptor mutation and hepatitis $E$ sero-positivity in liver transplant recipients. J Med Virol. 2020;1-4.

https://doi.org/10.1002/jmv.26236 